\title{
Hack the Experience: Scaffolding Codesign Processes for Organizational Innovation in Language Learning
}

\author{
Richard Medina \\ Center for Language \& Technology \\ University of Hawai' $i$ at Mānoa \\ rmedina@hawaii.edu
}

\author{
Stephen Tschudi \\ Center for Language \& Technology \\ University of Hawai'i at Mānoa \\ stephen.tschudi@hawaii.edu
}

\begin{abstract}
Hackathons are collaborative gatherings where participants address a challenge that touches on a shared concern or problem. The hackathon event model is increasingly adopted by diverse types of organizations that involve an equally diverse set of participants. In this case study, we report on an online hackathon event that was adapted for an educational organization. The event was offered as an opportunity to build community and enhance innovation skills for a group of undergraduate language students from across the United States. In contrast to technology focused outcomes, participants in the event focused on designing and prototyping experiences common in language learning. The case study presents the elements and dynamics of the hackathon through the lens of codesign to illustrate how specific stakeholder design activity can be scaffolded and facilitated to produce actionable design artifacts. These artifacts capture the design process that subsequently informs organizational innovations and product discovery.
\end{abstract}

\section{Introduction}

Product discovery initiatives are necessary for an organization to effectively target, evaluate, and resource technology innovations [1]. Innovations that are pursued should result in the intended impact for stakeholders and must be sustainable. Many projects fail to have this impact because they are not adopted by stakeholders, or they do not provide the functions actually needed, or they simply don't stick due to other external factors (E.g., changes in organizational leadership or broad shifts in enterprise-wide initiatives). No project is immune to these potential shortcomings but we can utilize methods for product discovery that can trend towards success rather than failure. There is strategic motivation for investing in product discovery processes within an organization. Pragmatically, we want to optimize allocation of resources for technology development projects, that is, we want to ensure that what we build is what is needed. Projects that are developmentally rooted in stakeholder investment and ownership lend themselves to more sustainable products and practices [2,3]. Organizational investment in design activity can be rewarded through adoption and potentially improved practices. One way to develop such roots is to involve stakeholders early in the product discovery phase, where what and why a tool should be built are the essential questions.

Engaging stakeholders early in the ideation and conception of product ideas provides insight into the practical views of their work by pursuing the question of "what is." Further, this early engagement may expose stakeholder views on potential transformation and improvement of those practices by simultaneously pursuing the question of "what could be" [4].

In this paper we present a case study on the design and adaptation of a hackathon event model as a mechanism for participatory design within a U.S. based educational organization focused on second language acquisition. ${ }^{1}$ The hackathon model was adopted to mediate a form of product discovery to identify and target organizational innovation within the program by involving a community of learner stakeholders in the ideation of future technologies for second language learning that may have tangible impacts on their second language development. We will view the hackathon model through the lens of codesign by highlighting the artifacts and design processes that supported participants during the event. This study also sheds light on the adoption of codesign methodologies within an educational technology setting.

The remainder of this paper provides a brief background on codesign, hackathons and the motivation

\footnotetext{
${ }^{1}$ The case study presented in this paper is drawn from a project conceptualized by and under the active direction of Dr. Julio Rodriguez, Principal Investigator of the Language Flagship Technology Innovation Center and Director of the Center for Language \& Technology at the University of Hawai'i at Mānoa.
} 
of the organization hosting the hackathon event. We then outline features of the hackathon model relevant to the case study then present the details of the event with a specific focus on design processes and scaffolded artifacts developed to elucidate collaborative ideation among participants [5]. We conclude with a discussion of this adapted model and its current and potential impact on the integration of codesign methodologies for educational technology innovation.

\section{Background}

\subsection{Participatory Design/Codesign}

Codesign is an activity that engages designers with stakeholders in the design of a product or solution. Its enduring characteristic, as a form of participatory design, is to facilitate authentic interaction between designers and the stakeholders most affected by the technology or product under consideration [6]. Approaches to codesign are varied by organization, the background of the designers, the make-up of stakeholders, the setting of the work, and the structure of the community of interest surrounding the work. The relationship can vary along a continuum, for example, with codesign activities that are designer-driven as opposed to activities that are driven by stakeholders but facilitated and advised by designers.

Sanders and Stappers [7], suggest a useful shared vocabulary for discussing codesign as a methodology for examining its application in practice. They suggest a typology of codesign activities that are categorized as probes, generative toolkits, and prototypes. Probes are activities that attempt to elucidate knowledge from stakeholders. For example, designer-led pre surveys or questionnaires that prompt for information or ideas related to the design objective. Generative toolkits are collections of tools that support stakeholders in expressing their ideas and perspectives through constructed representations. They can be driven by stakeholders. Examples of toolkits are collections of modeling primitives such as manipulable shapes and objects for showing differences, relationships, or dynamics. Prototyping is a type of activity that expresses a representation of a design that is accessible for testing and evaluation. Prototyping might typically be carried out by designers but stakeholders too can contribute to devising and evaluating prototypes. All three types of activity have a role for the stakeholder to fill. Degrees of agency across these activities may differ based on the skill set and orientation of both the stakeholder and the designer. Codesign activity elicits engagement from stakeholders where ideation, perspective sharing, meaning making, and solution finding are firmly grounded in the stakeholders' experiences, vocabularies, and skillsets. They can clearly express their practices through the mechanisms provided.

Alternatively, other forms of codesign elicit engagement with the added challenge that methods for ideation and perspective taking may not be as defined or developed equally among participants. The means for constructing artifacts of engagement and enabling expressive solutions through domain-specific formalisms can be relatively new or entirely unfamiliar to the participants. In some contexts, a useful generative toolkit requires more facilitation and support to be productive for the participants. One human-centered approach that facilitates codesign activities such as probing, making with toolkits, and prototyping in a variety of ways, yet is confronted with this dilemma is the hackathon event model [8][9].

\subsection{Hackathons}

Hackathons are organized gatherings of limited duration during which a group of people collaborate to develop solutions that address a shared problem or challenge [10]. Initially, they referred to a short two- or three-day event where computer programmers would gather to develop and implement software in response to a challenge. Hackathons, however, have emerged over the last decade as a more generally applied mechanism for collective ideation that reflects stakeholder engagement and investment in decision making [11]. These events are also intended to be fun and social. Some events are competitive, where solutions from teams are judged and a "best" solution is selected as a winner. Other hackathon events stress ideation and knowledge discovery and less so the production of a software solution (or "hack") for a particular challenge. Hackathons can be categorized as non-technical or technical with outcomes that can be tangible or intangible [12].

The hackathon event design has been co-opted and adapted for a wide variety of purposes and communities ranging from water preservation groups [13] to groups hoping to counter health and medical disinformation [14] where relatively short brainstorming and collaboration is seen as a productive way to discover or uncover new ideas or perspectives on a particular issue.

The intended outcomes designated for a particular hackathon are multifaceted. They can target solution finding while also building skill sets and stronger social ties within an organization. Hackathons can provide opportunities for training skills such as collaboration, design thinking, team building, and project management. They are also community building events where participants can strengthen communities of interest and establish social ties that may carry forward 
well past the event itself [13]. While the organization and dynamics of hackathons may vary by context, there are common parameters of a hackathon event model that are useful for evaluation and analysis.

The design of the hackathon described in this paper was shaped by the context specific parameters listed in Table 1. The goal was to seek innovative ideas from stakeholders while also facilitating skill development, community building, and the construction of an adequately articulated set of design prototypes. In the next section we present a case study of the hackathon to illustrate how the event design facilitated design processes for participants and contributed to organizational innovation in an educational setting. We propose two questions to structure our study.

Our first question is motivated by seeking to understand the efficacy of codesign activity. How can we ensure that the processes and deliverables of codesign activity are accessible and productive for the stakeholders and actionable by the organization? Our secondary question focuses on the event design itself. What specific properties of a hackathon should be targeted and evaluated for productive codesign activity? How are probes, toolkits, and prototypes mapped to the elements of the event? We are concerned with evaluating the event structure to optimize and improve subsequent hackathon events for the purpose of ongoing organizational and technology innovation.

\section{Case Study}

The case study described below was drawn from the "Hack the Language Flagship" hackathon event held in September 2020. The four-day online event was sponsored by The Language Flagship Technology Innovation Center (or "Tech Center") located at the University of Hawai'i at Mānoa. Table 1 summarizes the key elements of the event relevant for illustration in this paper. The following paragraphs describe in more detail how these hackathon elements were organized as a particular kind of codesign project. The first author was the project lead. Both first and second authors were involved in the planning and design of the event during the three months prior to the event. Both authors contributed to the conceptualization and planning of event activities, supporting documents and personally participated in the event as part of the panel of expert consultants and coaches. Data sources for the study are taken from event planning discussions and documents, direct observation of event activities, participants' digital artifacts captured during the event, preregistration information and post event survey responses. A case study approach is used to provide adequate detail for reflecting on and addressing the research questions proposed above by exhibiting the factors affecting productive codesign activity and its relation to properties of the event itself.

\section{Table 1. Hackathon summary}

Organization Tech Center mission is to enhance language learning development through integration of technology and organizational innovation.

Designers/Or
ganizers

Tech Center faculty and staff. Range of expertise that includes human-computer interaction, learning and instructional design, and world language education.

\begin{tabular}{|c|c|}
\hline $\begin{array}{l}\text { Expert } \\
\text { Consultants/ } \\
\text { Coaches }\end{array}$ & $\begin{array}{l}\text { World language educators, } \\
\text { administrators, and technologists }\end{array}$ \\
\hline $\begin{array}{l}\text { Stakeholders/ } \\
\text { Participants }\end{array}$ & $\begin{array}{l}23 \text { undergraduate students with } \\
\text { diverse majors and backgrounds }\end{array}$ \\
\hline Theme & $\begin{array}{l}\text { "Building Resilience in Language } \\
\text { Learners and Technology } \\
\text { Innovators" }\end{array}$ \\
\hline $\begin{array}{l}\text { Outcomes \& } \\
\text { Objectives }\end{array}$ & $\begin{array}{l}\text { Skill training, community building, } \\
\text { and product discovery }\end{array}$ \\
\hline Design Tasks & $\begin{array}{l}\text { Two design challenges: } \\
\text { 1. Design a VUCA activity } \\
\text { 2. Prototype VUCA activity } \\
\text { design as an enacted five- } \\
\text { minute film }\end{array}$ \\
\hline
\end{tabular}

Deliverables/ Activity design document and film Artifacts of enacted design specification

Format Four days with two challenge phases, six teams (3-4 persons/team), online

\subsection{About the Organization}

The Tech Center is a federally funded organization dedicated to language learning technology innovation in support of The Language Flagship program (or "Flagship"). The Flagship is a United States grant program that supports universities who wish to offer their students opportunities to gain high level language proficiency and intercultural competence in one of the several Flagship languages (Arabic, Chinese, Korean, Persian, Portuguese, and Russian). Students emerging from the Flagship program can function as global professionals, that is, people whose expertise lies not 
only in the specific subject area of their major, but also in their ability to perform professional-level work related to that major in the target language and cultural setting.

The Tech Center is a program within The Language Flagship initiative designed to incentivize and support innovation in language learning technology. Its organizational goals are to "identify, design, develop, deliver, and evaluate effective means to blend technology into existing Flagship programs" [15]. The Tech Center meets these goals through many different projects that interface with faculty and students across 31 Flagship programs in different ways. One such project that is specifically designed for Flagship students is the hackathon event discussed in this case study.

\subsection{Designers, Organizers \& Consultants}

The hackathon organizers and designers are made up of Tech Center faculty and staff representing a range of expertise in computer science, human-computer interaction, world language education, linguistics, and learning and instructional design. The organization's network is further expanded and enhanced with technology companies and academic consultants that work in the language technology field. The internal project team consisted of Tech Center faculty and staff while external consultants and partners were involved in the event as hackathon coaches (also called mentors).

\subsection{Participants}

Flagship students at each partner institution pursue a major field of study of their choice while concurrently developing proficiency in a second language also of their choice. Students within this program are highly motivated to enhance their professional careers by developing these second language skills. For the hackathon, students were the primary participants. Twenty-three students from seven different universities joined the event (Figure 2). Figure 1 illustrates the diverse fields of study that are pursued by participants. Despite a rather dispersed range of interests, student stakeholders share a common interest in language learning and technology as it relates to their personal investment in learning a second language.

\subsection{Theme}

The 2020 hackathon was based on the theme of "Building Resilience in Language Learners and Technology Innovators." Teams were assigned by the Tech Center based on commonalities analyzed in the registration materials. Challenges were judged using an analytic rubric. The entire hackathon was conducted in cyberspace. To minimize group fatigue, activities were distributed across four days instead of two, and the only time when all participants in the hackathon were together in Zoom video conference meetings was during the kick-off on day 1 and the wrap-up on day 4. All activities in between were performed and managed independently by each team. A team of coaches and organizers were available for consultation throughout the entirety of the event. They were slotted on a rotating schedule in the Slack application to maximize time availability and provide just-in-time access to participants.

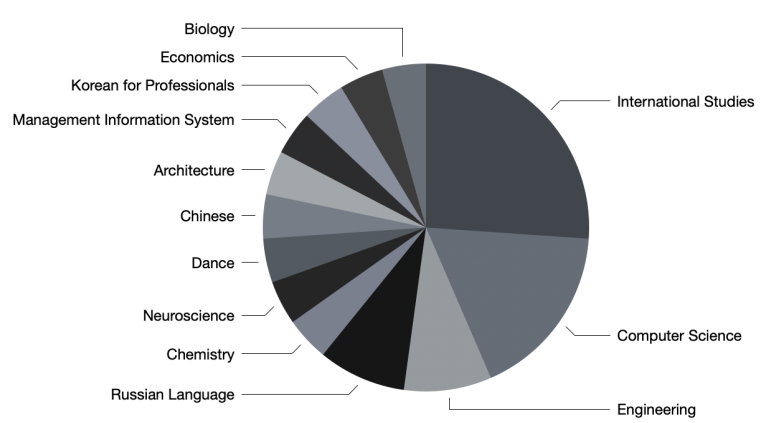

Figure 1. Participant major fields of study

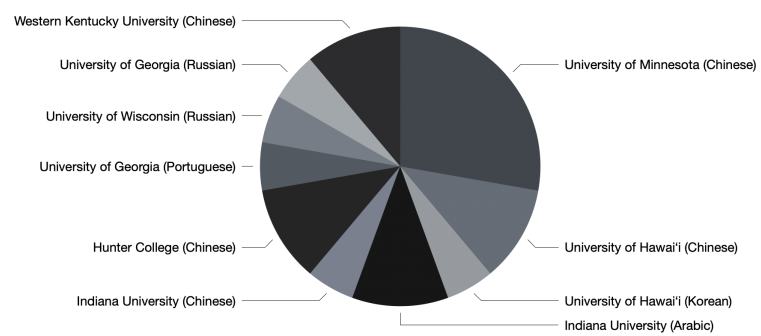

Figure 2. Participant sponsoring institutions

\subsection{Outcomes \& Objectives}

The structure of a hackathon meshes well with three of the organization's program goals. Expected outcomes for the organization were to enhance a community of innovators within the Language Flagship, develop collaborative skills in students applicable to their target profession, and to engage in innovative design processes. The objectives from the project team were to elucidate technology design ideas from the participants that addressed unique challenges that could be specified in a design document. In other words, a product discovery artifact. 


\subsection{Challenge I: A VUCA Activity Design}

The hackathon was organized around two successive design challenges over the four-day event. The first challenge was to specify an activity design (or game-like scenario) that reflected a volatile, uncertain, complex, and ambiguous (VUCA) environment or situation [16]. The rationale behind including this concept as a parameter of the activity was that second language learners often encounter situations that have these properties (E.g., culturally immersive situations that occur during study abroad residencies). Given this experiential familiarity, students were challenged with designing an interactive activity that, when implemented, would help the end-user develop skills for dealing with VUCA-like situations.

Table 2. Activity planning template
1. Title
2. Description
3. Goal
4. Obstacle
5. Activity Instructions
6. VUCA Skills
7. Learning Problem / Language Skills
8. Additional Materials (if any)
9. Activity Rubric
10. Dashboard Features
11. Dashboard User Narrative

To provide scaffolding for this design work, a starter kit containing detailed instructions and planning documents were issued to each team to use as guide during the construction of their VUCA activity design. Table 2 is a compact representation of a blank activity planning document participants used to design their activity. For each prompt, teams would identify how they would address each element of their design. Figure 3 is an excerpt from the participation guide on how participants might specifically target behaviors that would help a user cope with the VUCA environments. This additional information was provided to assist teams in addressing prompt 6 in Table 2. References to the "Dashboard" in prompts 10 and 11 (Table 2) are to a web application currently in development at the Tech Center. The Dashboard is a platform designed for hosting micro applications for language learning. It was intended to provide the teams a target context for the design activity as well as to impress upon them that the Tech Center intended to seriously consider taking up designs as formal projects. At the conclusion of the activity design challenge, each team submitted a design packet for scoring by a panel of faculty judges with expertise in instructional (activity) design. Each design packet included their planning document, their activity instructions, and a rubric for their activity. Of the six packets submitted for scoring, only the top three were selected to advance to the second challenge. Each of the three teams that were eliminated were merged into one of the teams that advanced. Figure 4 schematizes the structure of the hackathon with respect to challenge 1 and challenge 2 .

\begin{tabular}{|c|c|}
\hline Self-Awareness & $\begin{array}{c}\text { Flexibility / } \\
\text { Adaptability / Agility }\end{array}$ \\
\hline $\begin{array}{l}\text { Critical thinking, } \\
\text { reflection, receiving } \\
\text { and incorporating } \\
\text { feedback well. }\end{array}$ & $\begin{array}{l}\text { Changing quickly to } \\
\text { meet ongoing } \\
\text { changes in your } \\
\text { environment. }\end{array}$ \\
\hline Collaboration & $\begin{array}{l}\text { Creativity and } \\
\text { Innovativeness }\end{array}$ \\
\hline $\begin{array}{l}\text { Ability to make } \\
\text { connections with } \\
\text { others, } \\
\text { communicate well, } \\
\text { work in a team, and } \\
\text { problem solve. }\end{array}$ & $\begin{array}{l}\text { Ability to quickly } \\
\text { synthesize } \\
\text { information and } \\
\text { understand and } \\
\text { then disrupt the } \\
\text { status quo. }\end{array}$ \\
\hline
\end{tabular}

Figure 3. Strategies to cope with VUCA situations

\subsection{Challenge II: Prototyping a VUCA Activity}

The second challenge for the advancing teams was to demonstrate (or implement) the activity design from challenge 1 as a short film. Participants were instructed to provide an interpretation of the activity design through role play and scenario enactment in a short fiveminute film. This interpretive activity was intended to have teams prototype the activity design allowing participants to evaluate and assess elements of their design. The films were simulcast during the day 4 closing ceremonies where all participants (including audience members) voted on the winner.

\subsection{Deliverables}

There were two deliverables from the hackathon after day 4: a design packet containing a set of design documents that specified a VUCA activity and a short 
film enacting an activity for demonstration and evaluation.

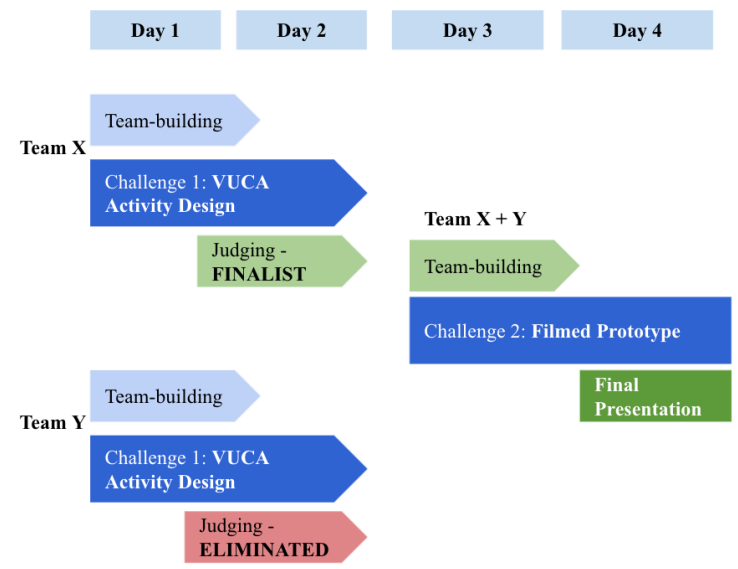

Figure 4. Hackathon event flow

Figure 5 illustrates a sample excerpt from the activity design document from one of the finalist teams. Their design focused on a writing exchange game with a clearly defined goal (develop versatile communication skills), an identified obstacle (avoid repeating vocabulary), and a set of activity instructions outlining how the activity is "played."

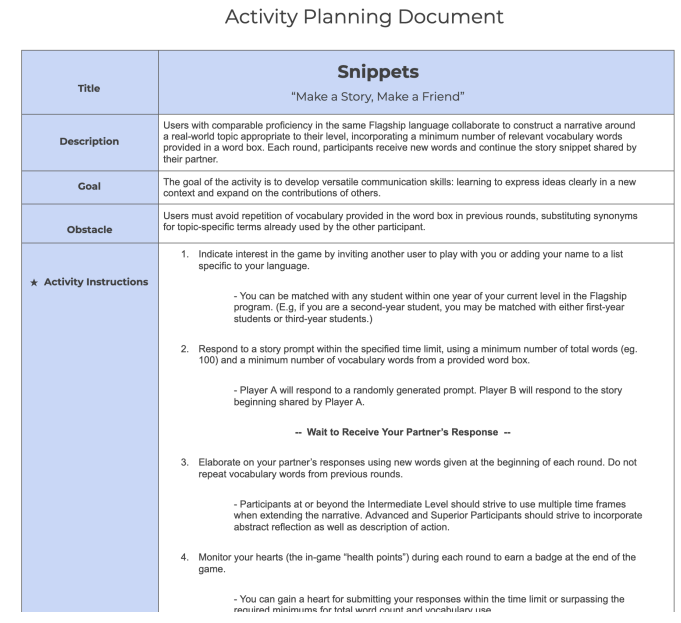

Figure 5. Excerpt from an activity planning document from one of the finalists

Figure 6 is a continuation of the activity planning document in Figure 5 showing the VUCA response strategy, a target learning problem or language skill (E.g., writing, interpersonal communication), a rubric for the activity, and integrations with the Dashboard platform.

Figure 7 is an excerpt from the activity instructions from the same team showing their construction of end-user facing documentation for the activity. The design documents including a rubric (not shown) were delivered as part of the design document packet. The second deliverable from the teams was the filmed enactment of the activity in a five-minute video. All videos are publicly available on the hackathon project site at Tech Center website.
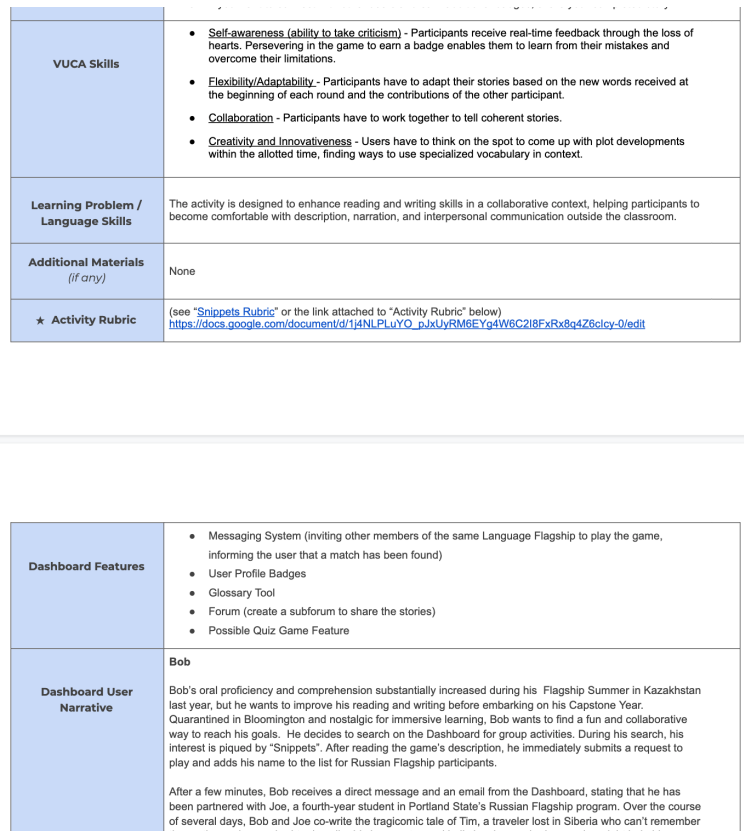

Figure 6. Excerpt from an activity planning document from one of the finalists (continued)

\section{Discussion}

A central planning challenge for hackathons at the Tech Center is accommodating the heterogeneous background of the students. The organizers could not presume all participants are familiar or even marginally skilled with specific tools or concepts related to technology design or design processes. Further, as noted above, participants are pursuing different majors and can be at different stages of matriculation within their respective institutions. Therefore, designing the hackathon challenges focused on identifying a frame of reference to plausible shared experiences and skill sets. The following discussion will focus on the engineered challenges and the related artifacts to address the research questions through the lens of codesign. In particular we will draw upon the vocabulary proposed by Sanders and Popper [7] to illustrate these dynamics. 


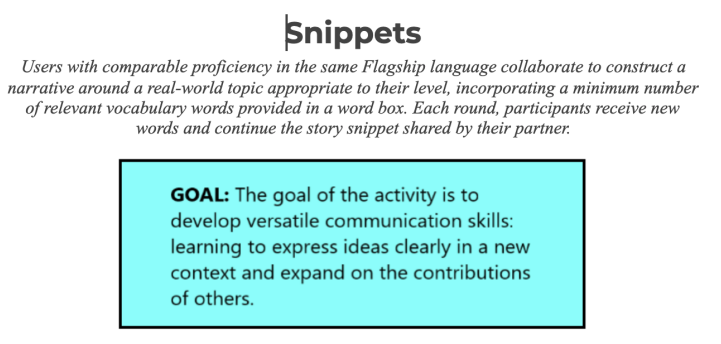

1. Matchmaking

Indicate interest in the game by inviting another user to play with you or adding your name to a list specific to your language.

NOTE: You can be matched with any student within one year of your current level in the Flagship program. (E.g, if you are a second-year student, you may be matched with either firstyear students or third-year students.)

\section{Begin the story}

Respond to a story prompt within the specified time limit, using a minimum number of total words (e.g. 100) and a minimum number of vocabulary words from a provided word box.

- Plaver A will respond to a randomlv generated prompt. Plaver B will

Figure 7. Excerpt from end-user facing instructions from one of the finalists (continued)

\subsection{How can we ensure that the outcomes and deliverables of codesign activity are productive for the stakeholders and actionable by the organization?}

To ensure that the codesign activities in challenge 1 were productive for the stakeholders, the organizers provided an extensively developed starter kit that provided instructions, necessary background information, and an activity planning document template for teams to structure their ideation. Of these, the activity planning template provided the clearest example of scaffolding each team's work to make certain elements of the design more salient [17]. The eleven indicators in Table 2 acted as prompts that were appropriated as a generative toolkit by providing a set of primitives from which the design activity could be constructed. All six teams in the hackathon submitted their activity design packets on time and fully completed. For the organizers, the result of having a total of six fully specified designs, all of which addressed critical elements of the intended activity, provided an invaluable record of the ideation that took place - a documented collection of detailed product ideas that are immediately available for further action.

The short film production activity in challenge 2 played a central part in the process of prototyping. A prototype facilitates a kind of "making" in which ideation remains a prominent function but its purpose is to evaluate and test a design. Video is an accessible media among the stakeholders and was chosen because it presented the least number of obstacles for expression and representation of interactive behavior intended in the activity designs. The film prototypes challenge was introduced to allow stakeholders the opportunity to enact and tell the story of their envisioned experience through a medium they were sufficiently familiar with. They had the necessary skills to productively produce the prototype. For organizers, the films provide access to the thoughts and motivations driving the design details, aspects that may not have been clearly expressed in the design document.

Collectively, these artifacts, one technical and the other expressive, contribute to an empathetic understanding of the stakeholders with respect to what they identify as important or worth pursuing. The activity planning documents give the designers an enduring structural representation of design details while the film exposes the nuance of the intended design. Through these artifacts, the authors, as designers, have a rich resource for understanding how stakeholders (language learners) perceive and cope with VUCA-like environments and situations.

\subsection{What specific properties of a hackathon should be targeted and evaluated for productive codesign activity?}

First, hackathon design should prioritize mechanisms that enable the formation of team autonomy as early as possible in the event. In the case discussed here, this autonomy was facilitated by careful construction of a generative toolkit and choice of prototype tool (video) to leverage as much as possible the skills that participants bring to the event. By choosing tools and materials that were familiar to participants, they were able to constructively and efficiently conduct (teams completed their challenges on time) their collaborative ideation. For example, coaches' availability, although meticulously scheduled to allow for time zone differences, was not utilized to the extent expected. Mentors generally play a substantive domain-specific role in hackathons that are face to face and are perceived as less effective when mentorship is provided remotely [9]. Despite this lessened reliance on mentorship to tackle both the activity design and film challenges, each team proceeded to complete creative projects.

Second, hackathon design should emphasize the activity of making [18]. The notion of constructing an artifact as a form of ideation has profound implications for understanding stakeholder views on their work, their practices, and their community. As discussed above, 
adequate scaffolding and guidance is key for facilitating authentic stakeholder generated artifacts from codesign projects. This dynamic becomes more critical in the context of a time constrained hackathon as described here.

Participant stakeholders produced quality and detailed activity specifications using seemingly mundane artifacts such as documents and video enactments. $93 \%$ of the 19 responses to our post event survey were satisfied. $70 \%$ of the participants indicated that challenge 1 was most enjoyable. From our analysis, this positive engagement was affected by carefully crafted support for design processes through scaffolded templates, clear explanation of terminology, instructions, and strategically selected tools for making and prototyping.

The quality and creativity of the ideas developed during the four-day event are evident in post hackathon initiatives. The "Snippets" design (illustrated above) is currently being prototyped for integration into a Tech Center application project. A number of the team members from that team also play a role in this extended and ongoing work as core contributors of the Dashboard Special Interest Group, a working group created to further engage students in the design activity generated by the hackathon event. (This working group is co-led by one of the authors.)

A product discovery process is evident here. Through the hackathon codesign event, designers are able to act on creative innovation from stakeholders. The activity design document is a technical artifact, a detailed and structured specification for a user experience that can be operationalized for further refinement by a design team. Together, with the related film, designers are exposed to insights into an expressed need and its intended solution or enactment.

As a mechanism for codesign and product discovery, hackathons require flexibility and openness for ideation on one hand and generation of technical artifacts and prototypes on the other. The challenge with this is the heterogenous background of the hackers/stakeholders and their potential unfamiliarity with the conceptual and technical domains required for efficient evaluation of expressed solutions. Importantly, the observations from the hackathon presented here suggest that generative toolkits that are scaffolded can accommodate both the authentic stakeholder perspective and tangible design artifacts needed to conduct product discovery.

\section{Conclusion}

The hackathon was a non-programming event. The caricature of hackathons as limited to "coders" (or hackers) is waning. In our view, the framing of codesign towards the human experience (with the tool) rather than the tool itself expands the source of organizational innovation from a broader set of stakeholders. Facilitating product discovery processes through codesign events, such as hackathons, can inform how an organization can converge on solutions that address the experiences of their community.

\subsection{Acknowledgements}

The authors wish to acknowledge Linchpin International as key contributors to the hackathon project while working under contract with the Language Flagship Technology Innovation Center.

The hackathon project (Hack the Language Flagship) was funded under a grant from the Institute of International Education (IIE), acting as the administrative agent of the Defense Language and National Security Education Office (DLNSEO) for The Language Flagship. One should not assume endorsement by the Federal Government. Project P.I.: Dr. Julio C. Rodriguez (juliocr@hawaii.edu), Center for Language \& Technology, University of Hawaii at Manoa.

\section{References}

[1] J. Münch, S. Trieflinger, and B. Heisler, "Product Discovery - Building the Right Things: Insights from a Grey Literature Review," in 2020 IEEE

International Conference on Engineering, Technology and Innovation (ICE/ITMC), 2020, pp. 1-8. doi: 10.1109/ICE/ITMC49519.2020.9198328.

[2] N. Linnell, S. Figueira, N. Chintala, L. Falzarano, and V. Ciancio, "Hack for the homeless: A humanitarian technology hackathon," in IEEE Global Humanitarian Technology Conference (GHTC 2014), 2014, pp. 577584.

[3] A. Nolte, E. P. P. Pe-Than, A. Filippova, C. Bird, S. Scallen, and J. D. Herbsleb, "You Hacked and Now What? -Exploring Outcomes of a Corporate Hackathon," Proc. ACM Hum.-Comput. Interact., vol. 2, no. CSCW, pp. 1-23, 2018.

[4] M. Steen, "Tensions in human-centred design," CoDesign, vol. 7, no. 1, pp. 45-60, 2011.

[5] J. van Dijk and R. van der Lugt, "Scaffolds for design communication: Research through design of shared understanding in design meetings," Artif. Intell. Eng. Des. Anal. Manuf., vol. 27, no. 2, pp. 121-131, 2013, doi: $10.1017 / \mathrm{S} 0890060413000024$.

[6] C. Spinuzzi, "The Methodology of Participatory Design," Tech. Commun., vol. 52, no. 2, pp. 163-174, 2005.

[7] E. B.-N. Sanders and P. J. Stappers, "Probes, toolkits and prototypes: three approaches to making in codesigning.," CoDesign, vol. 10, no. 1, pp. 5-14, 2014. 
[8] T. Zamenopoulos et al., "Types, obstacles and sources of empowerment in co-design: the role of shared material objects and processes," CoDesign, vol. 17, no. 2, pp. 139-158, 2021, doi: 10.1080/15710882.2019.1605383.

[9] A. Nolte, L. B. Hayden, and J. D. Herbsleb, "How to support newcomers in scientific hackathons-an action research study on expert mentoring," Proc. ACM Hum.-Comput. Interact., vol. 4, no. CSCW1, pp. 123, 2020.

[10] American Psychological Association, "What is a hackathon?," Psychological Science Agenda, vol. 2020, no. March, 2020. [Online]. Available: http://www.apa.org/science/about/psa/2020/03/hackat hon

[11] N. Taylor and L. Clarke, "Everybody's Hacking: Participation and the Mainstreaming of Hackathons," in Proceedings of the 2018 CHI Conference on Human Factors in Computing Systems, New York, NY, USA, 2018, pp. 1-12. doi: $10.1145 / 3173574.3173746$.

[12] M. A. Medina Angarita and A. Nolte, "What Do We Know About Hackathon Outcomes and How to Support Them? - A Systematic Literature Review," in
Collaboration Technologies and Social Computing, Cham: Springer International Publishing, 2020, pp. $50-64$.

[13] J. M. Carroll and J. Beck, "Co-designing platform collectivism," CoDesign, vol. 15, no. 3, pp. 272-287, 2019.

[14] N. Whitehead, "Ready, Set ... Think! Hackathon Aims To Kill Off Fake Health Rumors," Goats and Soda, Jun. 10, 2021.

https://www.npr.org/sections/goatsandsoda/2021/06/1 0/1004317823/ready-set-think-hackathon-aims-to-killoff-fake-health-rumors

[15] "The Language Flagship Program (online)." https://thelanguageflagship.org/content/educatorsadministrators

[16] S. A. Shambach, "Strategic leadership primer," ARMY WAR COLL CARLISLE BARRACKS PA, 2004.

[17] J. H. Larkin and H. A. Simon, "Why a diagram is (sometimes) worth ten thousand words," Cogn. Sci., vol. 11, no. 1, pp. 65-100, 1987.

[18] E.-N. Sanders, "Generative tools for co-designing," in Collaborative design, Springer, 2000, pp. 3-12. 